\title{
Study on Impact of Mobile Internet APP Interface Design to Brand
}

Zhang Haiyan

(Lanzhou Jiaotong University Art and design institute, Lanzhou, Gansu province

730070)

KeyWords: APP interface, brand propagation, basic elements, optimization design Abstract: After the creation of the mobile Internet, as a kind of mobile client terminal, APP is growing rapidly, even it has the trend to make the mobile Internet become the form of APP. In this era dominated by brand economy, as a kind of new propagation medium, APP makes the brand propagation become more diversified. The mobile Internet era is a We-media era, anytime and anywhere it all can create the propagation. However, it also puts forward the higher requirements for the brand. At present the visual homogeneity and the brand improper propagation of APP on the market emerge in endlessly. However, these are not helpful to create the brand effective propagation. At the same time, facing the increasingly fierce brand competition and the unceasing change of market requirement, APP also needs to constantly update and optimize with it. How to better promote the APP interface optimization design and realize the effective long-term development of brand propagation is the emphasis of this paper.

\section{Introduction}

With the popularity and promotion of mobile Internet, the development of mobile devices, such as mobile phone, computer, tablet computer and other hardware technologies, the user group development of mobile terminal develops faster and faster. APP is the function application on the mobile devices. Today the user group becomes more and more huge, in the future the mobile Internet even has development trend of becoming the form of APP. Brand reveals the image and the power of an enterprise, at the same time, the enterprise also can improve its own superior power by increasing the brand value. Expand the market, and grab market share. As far as APP is concerned, the APP interface design is the most intuitive display of its brand image. If we want to make a successful APP, the interface design is crucial. 


\section{APP Interface Design Style and the Forming Reasons}

APP interface design produces the very big difference on the design concept and presentation mode, because affected by regional culture, economic development degree, thinking mode and other factors. In the globe with the population of nearly more than 7 billion, people's requirements of different nationalities, countries and regions are also different. In addition, people's requirements hierarchies are different. Even though the same person is at different times, the requirements are also different.

Speaking from the large group of regional decentralization, aiming at the target users in different regions the overall style of APP in the interface design will be different. As shown in Figure 1, Finland's APP interface design embodies the natural style of modern design, and Finland's designers are good at finding the inspiration from the nature. In 2014 Finland National Tourism Administration

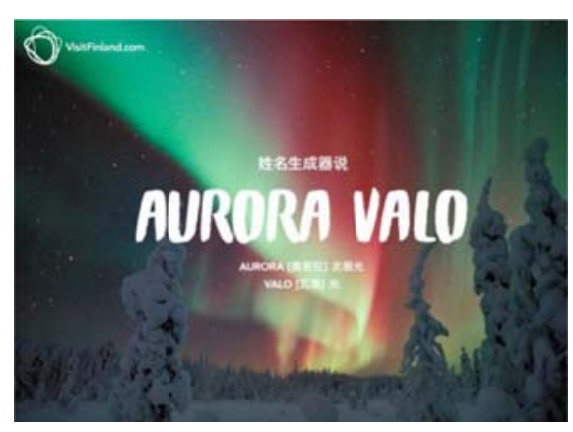

Figure 1 "Finngenerator" APP launched the mobile terminal "Finngenerator" APP(Finland's names generator), and it set off a frenzy. It is said that this APP uses Finland's natural landscapes, animals, Finland national epics and other elements to make their own Finland's names be tailored to the users. This activity has attracted more than 1.7 million people to participate in exploring their own Finland's names, and have the opportunity to know the meaning behind the Finnish to let people have the opportunity to explore their own inner Finland elements so as to know this distinctive faraway country. Application method: If we input "Zhang Ziyi”, we can obtain the Finnish name "Saan Ahtisaari". Saan is Finland's most famous and magnificent mountain, and Ahtisaari means the island of Ice God.

The design of Denmark APP is simple and has lasting appeal, pays attention to user experience and takes people as the orientation. In 2015, Danish developers and non-profit organizations cooperated and launched "Be My Eyes" APP. As shown in Figure 2, this kind of APP seeks help from the masses. Through the simple video calls, it can help the blind persons to identify what the thing in front of our eyes are. The concept of this kind of APP is very simple. It makes

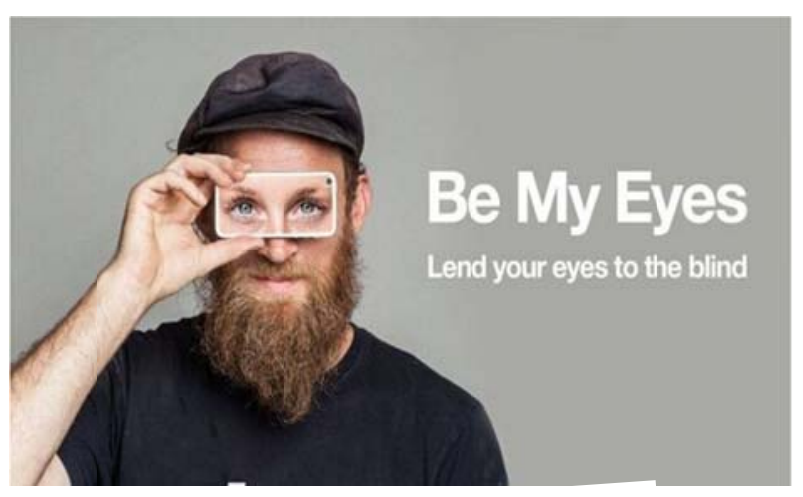

Figure 2 Be My Eyes APP the people with the visual impairment be paired with the people with normal vision. As long 
as the vision-impaired people take out of the mobilephones to record the texts which they need to identify or read. The warm-hearted people with normal vision can help them to read the things shown in the lens.
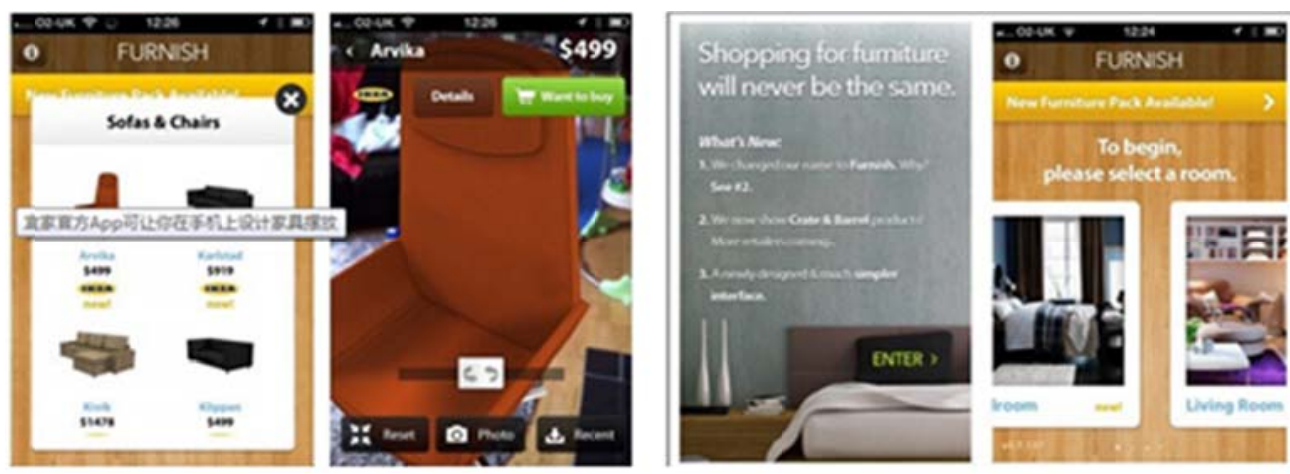

Figure 3 IKEA Now APP

Japanese design has its unique national style, because its design is based on national culture. With the constant practice for a long time it has formed its own standard on national aesthetics, and at the same time it also has reached the mature and refined level. The thing completed by the Japanese designer is not only the product design but also a kind of expression of national culture. In addition to paying attention to the traditional design style, Japanese APP also attaches much importance to the usages of color, graphics and fonts. It is shown in the following Figure 4 PETATTO CALENDAR APP.

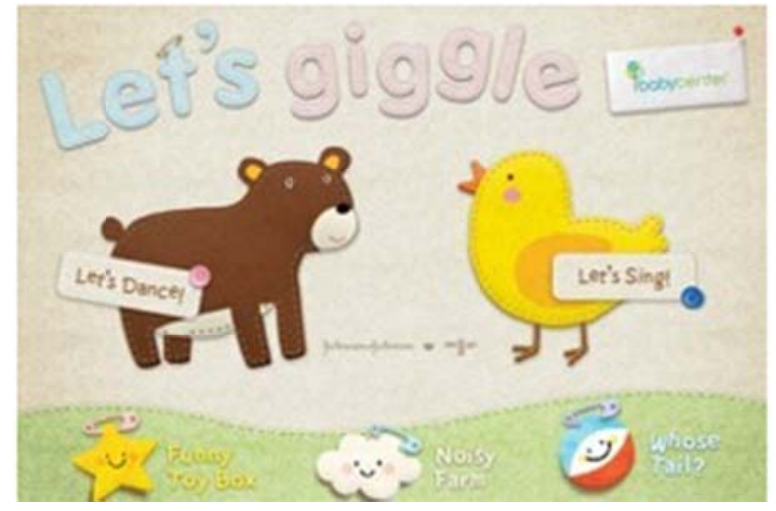

Figure 4 Let's Giggle APP

Because the style is a very broad visual concept, the analysis mentioned above may be just a small portion of APP design style differentiation. Because it is divided from the large 
group, the consumers will be affected by different regionalisms, different cultures, economic development levels, living habits, thinking modes and other different factors. Because it is divided from the small group, the consumers will be connected with age level, gender, specific major and other factors. The formation of style can be have very complicated reason, but we can start with the consumer requirement, and by classifying the consumers have insight into the requirements of target consumers so as to have the style orientation.

\section{Relationship between APP Interface Optimization Design and Brand Propagation}

Once a test showed that the first impression of human on the things would be completed within 50 milliseconds. In this fairly short time, the conveyance of APP interface design to brand image is crucial ${ }^{[18]}$. This is mainly affected by the primacy effect, and the influence of the first impression obtained can even affect the overall impression more than the subsequent impact. If the first impression is not good, it is difficult to obtain the user's approval behind. A beautiful design style and standardized element display is more beneficial to good interaction between users and application programs so as to better set up the brand image and obtain more effective brand propagation effect.

The significant economic benefits brought by brand economy urge the merchants to pay more and more attention to the spread of brand. The requirement of brand propagation urges the merchants to optimize the product design continuously. Even though we don't exclude some merchants' propaganda with the bombing type and in a short time the propagation range may become wide, it is difficult to obtain the consumer identity. In the long run, a good brand image can promote the effective propagation of brand, and the brand image is also a constant change and upgrading thing, then we need to continuously optimize and design it.

APP interface design needs to adapt to very strong characteristics of the Internet era, such as individual requirement, fragmented time, uninterrupted link, etc. About the optimization design we need to start from the user requirement, and pay attention to the user experience and usage scenario. While it is not just simple picture quality, sorting order and other simple adjustment and change. The so-called interface design, refers to that we need to conduct good human-computer interaction and visual induction.

\section{Optimization Design Analysis of Interface Design Visual Basic Elements}

\subsection{Style}

In the fierce era of brand competition, the style is even the important element of brand 
identity. However, at the same time, the style is a rather broad concept, and it even pays more attention to the unified expression of product character. First of all, according to the brand concept, we position the product character. For example, we take the cordial and naughty small fresh as the product character of Taobao APP to let the user enjoy the relaxed atmosphere while shopping. In interface design we can use interesting cartoon and lively painting style, and the copywriting can also very humorous. However, Amazon's style can be more practical. The science and technology feeling is relatively sufficient. The cartoon images and pictures can also rarely appear, because these are all related to the product brand character. As shown in Figure 5, NIKE is a brand which pays much attention to the product character expression. It has the velocity-sensing pictures with stimulation sense and the copywriting with incentive nature. These all reflect that the product is a professional international brand. These brand concepts may not be written directly, but they convey to the users' subconsciousness through some clever elements intentionally or unintentionally.

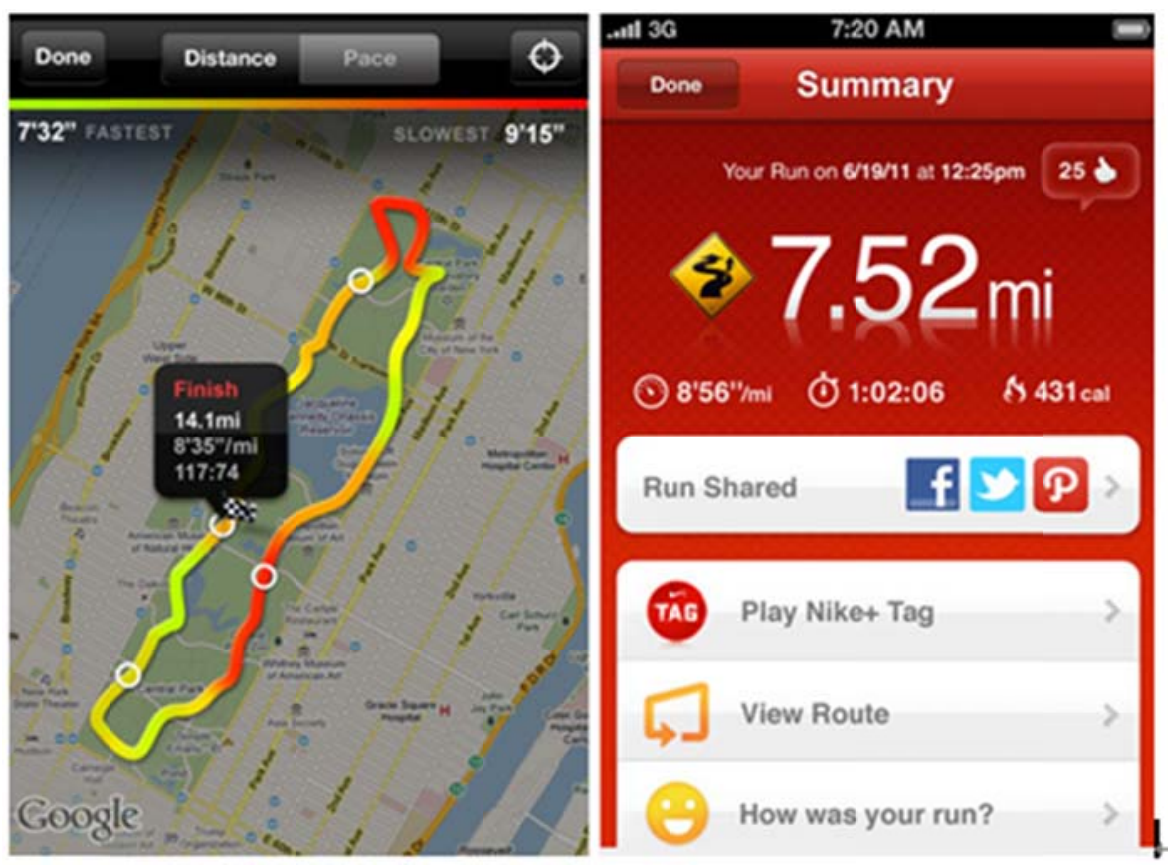

Figure 5 NIKE+ APP

\subsection{Layout}

The limited space on mobile screen has put forward some interesting restrictions for the effective presentation of content and interaction. Specifically speaking, the layout must be simplified and concentrated. Information layout is different in the interface, and it can 
affect the efficiency of user's access to the information. We can say that the format layout is a very important element in the interface design. The format layout structured by the placed location of visual elements can influence its important degree in the interface and have the direct impact on structural layout.

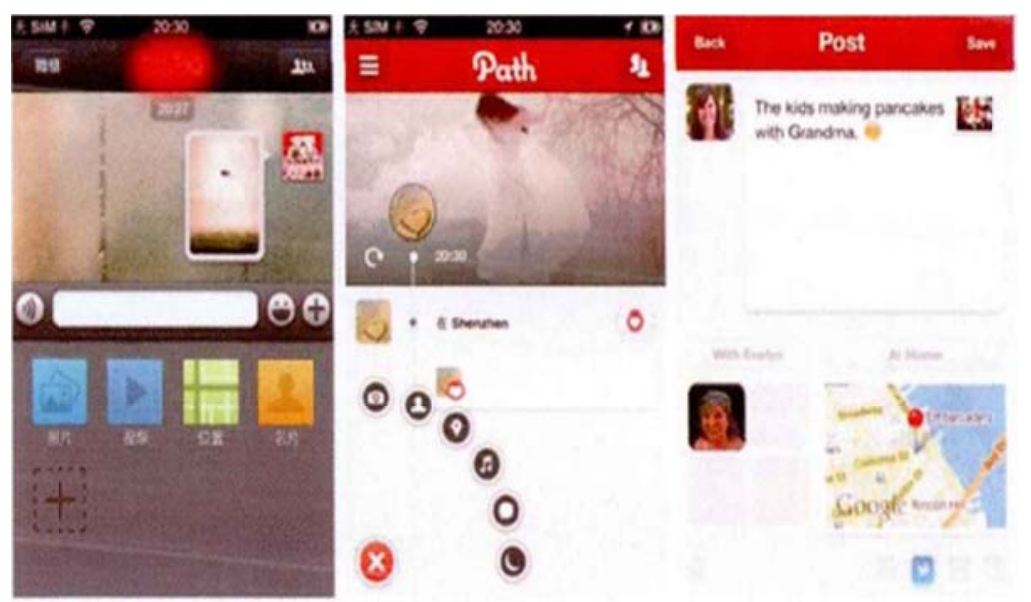

Figure 6 Path APP

\subsection{Color}

Color can help the brand to build the users' cognition very easily. Color is one of the main means used by each successful product. The consumers' purchasing process can produce brand propagation, and consumers' buying decision can have an effect on what kind of brand we can purchase. In the early 1980s, CMB companies in the United States have summarized in the rich marketing practice that we make use of color combination to promote the product sales. As shown in the following figure, yellow attracts attention and window shopping. Red has the stimulation and the sense of urgency, and it is often used to clearance sale. Blue emphasizes calmness and security, and it is often used in banks and business institutions. Green lets people relax and relieve stress, such as credit card repayment button. Orange has strong call meaning, and it is often used in creating, buying selling and other activities. Pink is suitable for women and it has the romantic tone. Black is the best matching of luxuries. Purple has quiet and soothing meaning, and it is often used in beauty and health products industry. 


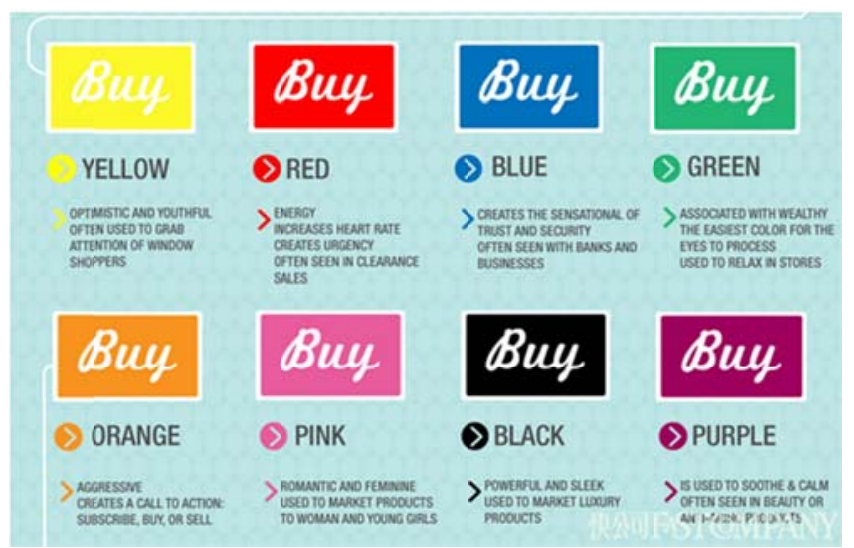

Figure 7 Colorful Buy Buttons

\section{Development Trendency of APP Interface Design under the Brand Propagation}

\subsection{Personalization}

Based on the interactive mode of consumer guide principle and mobile Internet consumption in brand propagation, the users even more hope to have a personalized service, and the brand also should propagate accurately. In APP interface design, taking the users as the center is an important design requirement. For both the creative concept and the concrete execution, the designers can't simply start from their own angle, otherwise it is easy to produce the "bad" user experience.

As shown in Figure 8, "Cute face" is the APP based on content which is filled with interesting things. Through it the users can customize their own personalized images. Due to its novel form and interesting content, it is sought after by many people.

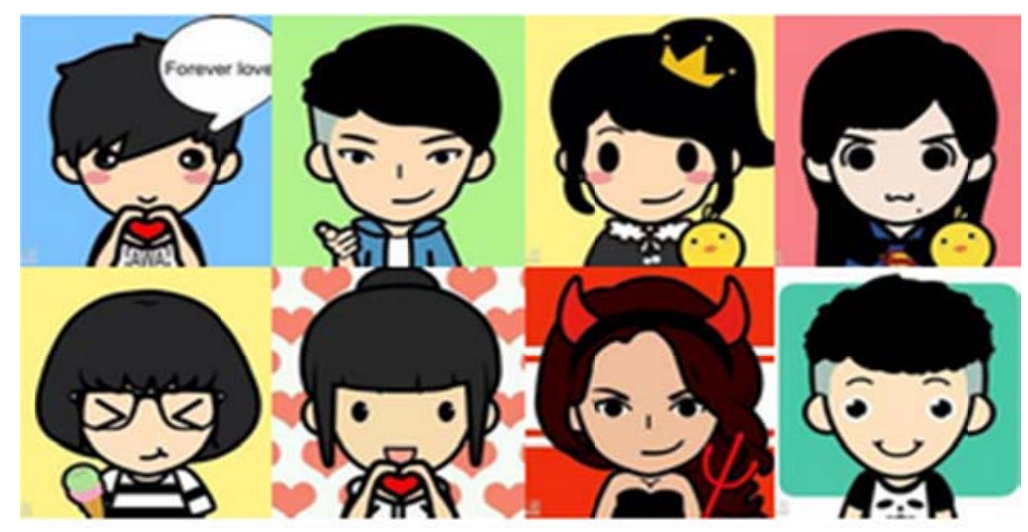

Figure 8 Cute Face APP 


\section{Conclusion}

In this mobile Internet era, APP acts as the expansion of mobile device functions and it starts to become an indispensable part in people's lives. The Internet age is a We-media era, anytime and anywhere it all can create the propagation. However, it also puts forward the higher requirements for the brand. Under the era background of brand economy, brand propagation seems to be critical, however, brand propagation can not be separated from the molding of brand image. As a part of the brand design, APP interface design plays a very important role in effective propagation of brand. APP interface design mainly includes the three parts, namely user research, interaction design and visual design. We mainly aim at the visual design part to conduct the study and put forward how to optimize and design it in the paper. However, the analysis on user research and interaction design aspects is not very systematic and in place. But if we want to a good APP product, the relationship between the three should be interrelated and promoted mutually.

\section{References}

[1]Huang Xiao. Ebook App Interface Design Research based on Interactive Theory[D]. North China University of Technology, 2015.

[2]Li Ting. Smartphone App Interface Design Research based on User Experience[D]. Taiyuan University of Technology, 2015.

[3]Zhao Xin. GUI Warming Design Research[D]. Xi'an Academy of Fine Arts, 2015.

[4]Huang Guanglei. Interface Design of Elderly Health Care Products[D]. Zhejiang Sci-Tech University, 2015. 